\title{
The British Columbia Healthy Connections Project: findings on socioeconomic disadvantage in early pregnancy
}

\author{
Nicole L. A. Catherine ${ }^{1 *}$ (D), Rosemary Lever ${ }^{1}$, Debbie Sheehan', Yufei Zheng ${ }^{1}$, Michael H. Boyle²,
} Lawrence McCandless ${ }^{3}$, Amiram Gafni ${ }^{4}$, Andrea Gonzalez ${ }^{2}$, Susan M. Jack ${ }^{5}$, Lil Tonmyr ${ }^{6}$, Colleen Varcoe ${ }^{7}$, Harriet L. MacMillan ${ }^{8}$, Charlotte Waddell ${ }^{1}$ and For the British Columbia Healthy Connections Project Scientific Team

\begin{abstract}
Background: Maternal exposure to socioeconomic disadvantage increases the risk of child injuries and subsequent child developmental and mental health problems - particularly for young mothers. To inform early intervention planning, this research therefore aimed to describe the health and social adversities experienced by a cohort of girls and young women in early pregnancy in British Columbia (BC), Canada.

Methods: Participants were recruited for the BC Healthy Connections Project (BCHCP), a randomized controlled trial examining the effectiveness of Nurse-Family Partnership, a home visitation program, in improving child and maternal outcomes. Baseline data were collected from 739 participants on trial entry. Participants were selected on the basis of preparing to parent for the first time and experiencing socioeconomic disadvantage. Analyses involved descriptive statistics and age-group comparisons.
\end{abstract}

Results: Most participants reported having low income (84\%), having limited education (52\%) and being single (91\%) at trial entry. Beyond these eligibility criteria, other health and social adversities included: housing instability (52\%); severe anxiety or depression (47\%); other diagnosed mental disorders (22\%); prenatal nicotine and cannabis use (27 and 21\%); physical health problems (20\%); child maltreatment when younger (56\%); and intimate partner violence recently (50\%). As well, few (29\%) had received income assistance entitlements. More than two thirds (70\%) were experiencing four or more forms of adversity. Age-group differences were observed for cognitive functioning, being single, low income, limited education, psychological distress and service use ( $p$-value $\leq 0.05$ ).

Conclusions: This cohort was selected on the basis of socioeconomic disadvantage. Yet all participants were experiencing substantial added adversities — at higher rates than other Canadians. Furthermore, despite Canada's public programs, these pregnant girls and young women were not being adequately reached by social services. Our study adds new data to inform early intervention planning, suggesting that unacceptably high levels of socioeconomic disadvantage exist for some young British Columbians. Therefore greater health and social supports and services are warranted for these young mothers and their children.

Trial registration: Registered August 24, 2012 with ClinicalTrials.gov Identifier: NCT01672060. Active not recruiting.

Keywords: Pregnancy, Adolescents, Maternal health, Socioeconomic disadvantage, Cumulative disadvantage

\footnotetext{
* Correspondence: nicole_catherine@sfu.ca

${ }^{1}$ Children's Health Policy Centre, Faculty of Health Sciences, Simon Fraser

University, Room 2431, 515 West Hastings Street, Vancouver, BC V6B 5K3,

Canada

Full list of author information is available at the end of the article
}

(c) The Author(s). 2019 Open Access This article is distributed under the terms of the Creative Commons Attribution 4.0 International License (http://creativecommons.org/licenses/by/4.0/), which permits unrestricted use, distribution, and

reproduction in any medium, provided you give appropriate credit to the original author(s) and the source, provide a link to the Creative Commons license, and indicate if changes were made. The Creative Commons Public Domain Dedication waiver (http://creativecommons.org/publicdomain/zero/1.0/) applies to the data made available in this article, unless otherwise stated. 


\section{Background}

Socioeconomic disadvantage poses challenges to the wellbeing of both mothers and children [1-3]. In particular, adolescent mothers (aged 19 years or younger) are more likely to experience interrupted education, lower workforce participation, lower income, unstable housing, and associated physical and mental health and cognitive problems [4,5]. Children born to adolescent mothers, in turn, are at greater risk for preterm birth, childhood injuries and subsequent developmental and mental health problems [6-8]. Similarly, children born to young mothers (aged 20-24 years) who are experiencing socioeconomic disadvantage (such as having low income, having limited education or having limited social supports) are also at greater risk for injuries and subsequent developmental and mental health problems [2, 9-12]. Other health and social adversities associated with maternal socioeconomic disadvantage in general include depression, prenatal substance use and exposure to intimate partner violence (IPV), which also adversely influence the developing child [11, 13-15].

Yet the socioeconomic disadvantage that underlies many childhood mental and physical health problems is socially produced and therefore may be amenable to intervention [16-18]. Providing children with a better start in life, beginning before or during pregnancy and continuing in the early years, promotes healthy development and results in greater societal benefits compared to later remediation of health and social problems [18-22]. It is therefore crucial to identify opportunities for intervening "upstream" - well before avoidable adversities occur and subsequent health and social problems begin.

One approach is to identify disadvantaged populations in early pregnancy and examine how avoidable adversities may be offset or muted by specific prevention interventions aimed at improving the life course trajectories for both children and mothers [18-22]. Reducing socioeconomic disadvantage and improving parenting through providing better supports for pregnant girls and young women and new mothers - is a powerful mechanism for supporting healthy development throughout the lifespan [1, 23]. Yet data describing populations of disadvantaged young mothers-to-be in Canada have been limited, in turn, limiting the data available to inform the development and provision of effective maternal and child services that are proportionate to the level of disadvantage or need [18]. As well, disadvantaged groups have often been characterized as "hard-to-reach" rather than "need-to-reach," further hampering intervention efforts [24]. To inform intervention planning, this research therefore aimed to describe the health and social adversities experienced by a cohort of 739 pregnant girls (14-19 years) and young women (20-24 years) in British Columbia (BC), Canada.

\section{Methods}

\section{Study design}

We report on baseline data from the $\mathrm{BC}$ Healthy Connections Project (BCHCP), a randomized controlled trial (RCT) evaluating the effectiveness of the NurseFamily Partnership (NFP) program compared with existing health and social services [25]. NFP involves nurses providing intensive home visits with young, low-income, first-time mothers, starting in early pregnancy and continuing until children reach age 2 years [23]. Developed in the United States, NFP has been shown to reduce child injuries and improve children's mental health and development, while also improving mothers' life circumstances, especially for those experiencing the highest levels of disadvantage [23]. We also compared data across both age groups (14-19 and 20-24 years) to ascertain similarities or differences in experiences of disadvantage in early pregnancy, and to determine whether we in fact had reached those whom NFP is most intended to help.

\section{Participants}

We used baseline (pre-randomization) data for 739 participants enrolled in the BCHCP. Participants were eligible if they: were in early pregnancy (less than 28 weeks gestation); preparing to parent for the first time; were young (24 years or younger); and were experiencing socioeconomic disadvantage, (a risk factor for child injuries, the trial primary outcome indicator). Indicators of disadvantage included: having low-income (receiving income assistance, or experiencing homelessness, or finding it very difficult to live on total household income with respect to food or rent); having limited education (less than high school); or preparing to parent while single (not married or not living common-law for one year or more). Pregnant girls aged 14-19 years were deemed to automatically meet disadvantage criteria due to their young age; young women aged 20-24 years were required to meet two of three indicators. Previous NFP trials in other countries enrolled pregnant girls living with low income [26], or girls and young women (less than 26 years old) experiencing disadvantage [27].

Referrals came from public health units at four regional BC Health Authorities (Fraser, Interior, Island and Vancouver Coastal Health). Recruitment targets were met after three years (that is, $60 \%$ were reached by public health nurses, of which $60 \%$ were enrolled, which comprised one third of all potentially eligible participants). Baseline data were gathered during in-person research interviews conducted in participants' homes between October 2013 and December 2016. Detailed trial information is described in the RCT study protocol [25]. Figure 1 shows participant flow. The BCHCP trial 


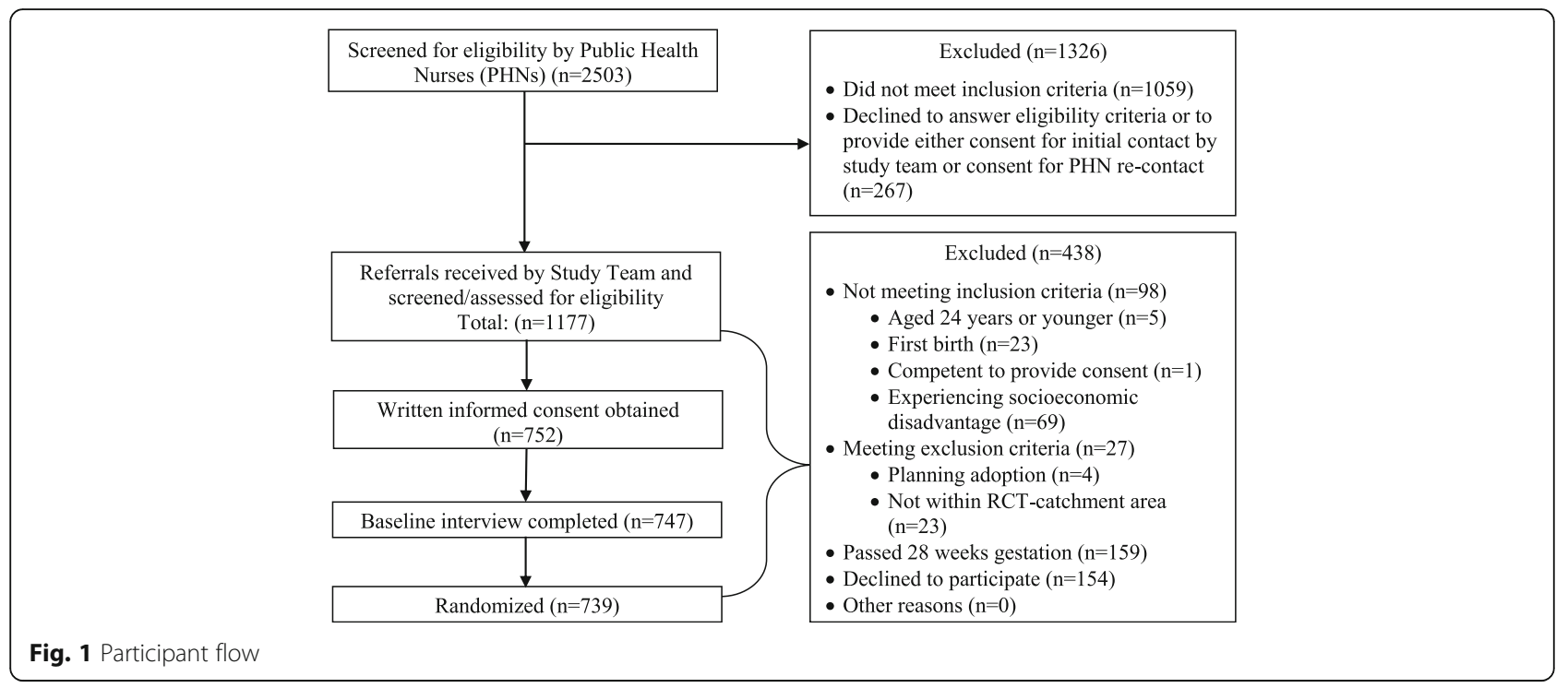

adheres to Consolidated Standards of Reporting Trials guidelines.

\section{Measures}

In the home interviews, participants confirmed that they met eligibility criteria. They also described their cultural background, first language and housing situations. As well, a comprehensive array of validated scales and items were administered covering: additional health and social adversities (housing, mental health including prenatal substance use, physical health, history of maltreatment as a child, exposure to IPV in the past year); receipt of health and social services; maternal psychological resources (self-efficacy, mastery); and maternal cognitive ability and executive functioning. To enhance accuracy, field interviewers verbally administered questionnaires and cognitive tests in-person. Sensitive items deemed prone to reporting bias (such as prenatal substance use) were confidentially administered using headphones with audiotaped questions; participants then placed written responses in sealed envelopes for later processing by the study team. See Table 1.

\section{Cumulative disadvantage}

The proportion of participants experiencing between one and eight indicators of disadvantage was calculated. Indicators included: living on low income (less than $\$ 20$, 000 annually CAD); having limited education (less than high school); preparing to parent while single; experiencing unstable housing (having to move three or more times or experiencing homelessness in the past year); experiencing moderate/severe levels of psychological distress; having any prenatal substance use in the past month; having been maltreated as a child; and experiencing IPV within the past year.

\section{Statistical analyses}

Descriptive statistics were used to characterize all variables. The data were screened for: accuracy of entry; patterns of missing data; and assumptions of normality, independence and homoscedasticity. To compare the two age groups (14-19 versus $20-24$ years), we used the Chi-square test (or the Fisher's exact test for cell sizes less than five). For continuous variables, we utilized the Student's $t$-test. Statistical significance was set at $p$-value $\leq 0.05$. In each table, $n$ may be different from $N$ due to missing data (for example, participants could choose not to respond to given items).

\section{Results}

Data are provided on the total cohort as well as on the two age groups in Tables 2, 3, 4, 5 and 6. Missing data were minimal at less than $2 \%$ for all variables, except for unstable housing (3\% missing) and English as a first language ( $7 \%$ missing). Central descriptives on the total cohort and statistically significant age group differences are summarized below.

\section{Baseline socioeconomic disadvantage according to eligibility criteria}

Nearly half of participants (49\%) were aged $14-19$ years, while just over half (51\%) were aged 20-24 years. Most of the cohort (84\%) were preparing to parent while living on low income (less than $\$ 20,000$ CAD annually); more than half (52\%) had not completed high school (including 182 pregnant girls or $25 \%$ of the total cohort who were still attending high school); and almost all (91\%) 
Table 1 Summary of measures

\begin{tabular}{ll}
\hline Measurement Construct & Description \\
\hline Sociodemographic characteristics & \\
& $\begin{array}{l}\text { Age, marital status, cultural background, first language, } \\
\text { education, income and housing. Income was defined } \\
\text { as pre-tax annual income from all sources of } \\
\text { employment including unreported income and } \\
\text { excluding any money received from family, friends or } \\
\text { income assistance [28]. }\end{array}$
\end{tabular}

Scoring

Psychological resources

\author{
Self-efficacy \\ Mastery \\ General Self-Efficacy Scale [29]. Likert scale 10 items, \\ e.g., "I am certain that I can accomplish my goals." \\ Pearlin Mastery Scale [30]. Likert scale 7 items, e.g., \\ "I have little control over the things that happened to me."
}

Cognitive ability

Executive functioning

Inhibition of interference

Visual attention and task switching

\section{Socioeconomic disadvantage}

Having low income

Having limited education

Being single (having limited social supports)

Homelessness

Unstable housing

History of child maltreatment

Child maltreatment age

16 years or younger

Exposure to intimate partner violence

Including physical abuse, emotional abuse and harassment

Mental and physical health

Psychological distress

Mental and physical health conditions

Prenatal substance use

Nicotine, alcohol, cannabis, and

Having to move three or more times or experiencing

Any long-term health conditions diagnosed by a physician affecting day-to-day activities $[40,41]$.

Frequency of use [42].
Shipley-II [31]. Vocabulary Subscale 40 items assesses acquired knowledge. Abstraction Subscale 25 items assesses abstract reasoning.

Stroop Colour and Word Test [32]. Cognitive assessment of ability to inhibit interference in the reaction time of a task.

Trail Making Test $[33,34]$. Participants were timed while first sequentially connecting numbered circles (1-2, 2-3, etc.; TMT-A), then lettered and numbered circles (1-A, A-2, 2-B, etc.; TMT-B).

Pre-tax annual income from all sources of employment including unreported income and excluding any money received from family, friends or income assistance.

Not completing the equivalent of a $\mathrm{BC}$ high school diploma.

Not married or common-law (living together consecutively for one year or more).

Living on the streets or in an emergency or homeless shelter, staying in places not meant as residences, (e.g., car or tent), and/or experiencing "hidden homelessness", (e.g., staying with someone because of no permanent address or having nowhere else to live or "couch-surfing") $[35,36]$. homelessness (past year).

Childhood Trauma Questionnaire - Short Form [37]

Likert scale 28 items, e.g., "When I was growing up,

I didn't have enough to eat."
Descriptives.

Higher scores represent higher levels of self-efficacy.

Higher scores represent higher levels of mastery.

Higher total raw scores indicate better performance.

Higher raw scores represent better cognitive performance.

Shorter times represent better scores. A ratio of TMT-B / TMT-A represents executive control [35].

Living on low income at $<\$ 20,000$ annual employment income.

Dichotomous (yes/no) variable.

Dichotomous (yes/no) variable.

Dichotomous (yes/no) variable.

Dichotomous (yes/no) variable.

Moderate-to-severe levels of any type of abuse or neglect.

Higher scores indicate higher levels of abuse.

Total scores of $>25$ represent moderateto-severe anxiety or depression.

Number and type of diagnosed conditions.
Dichotomous (yes/no) variables. 
Table 1 Summary of measures (Continued)

\begin{tabular}{lll}
\hline Measurement Construct & Description & Scoring \\
\hline \multicolumn{1}{c}{ other street drugs } & & \\
$\begin{array}{c}\text { Receipt of health and social services } \\
\text { Health services received }\end{array}$ & $\begin{array}{l}\text { Visiting primary healthcare providers } \\
\text { for physical concerns }\end{array}$ & Number and type of services. \\
& receiving prenatal classes. & Number and type of services. \\
Social services received & Income assistance through provincial or & \\
& federal programs such as: BC Income and & Disability Assistance, Canada Disability \\
& Benefits and Employment Insurance, BC Hardship & \\
& Assistance, and BC Youth Agreements. & \\
\hline
\end{tabular}

were preparing to parent while single. Compared to young women, more girls reported living on low income and having limited education, but fewer were preparing to parent while single. See Table 2.

\section{Sociodemographic characteristics}

Most participants (57\%) identified as "white", while over a quarter (27\%) identified as Indigenous (including First Nations, Métis or Inuit) and others identified as mixed heritage (7\%), Asian (4\%) or other cultural backgrounds (5\%). Most (93\%) reported English as their first language (in keeping with eligibility criteria requiring conversational competence in English). See Table 3.

\section{Psychological resources and cognitive functioning}

The mean raw scores for self-efficacy, mastery, cognitive ability and executive functioning are presented in Table 3. Compared to girls, young women had significantly better performance on measures of cognitive ability (vocabulary) and executive functioning.

\section{Health and social adversities including maltreatment experiences}

Participants experienced health and social adversities beyond those associated with the eligibility criteria including: lifetime homelessness (47\%); housing instability (52\%); moderate/severe psychological distress (32\%); severe anxiety or depression (47\%); other diagnosed mental disorders (22\%); prenatal nicotine and cannabis use (27 and $21 \%$ respectively); physical health problems (20\%); child maltreatment when younger (56\%); and exposure to IPV recently (50\%). More young women (36\%) also reported moderate/severe psychological distress compared to girls (28\%). See Table 4.

\section{Receiving health and social services}

Most participants (77\%) reported visiting primary healthcare providers (physicians, nurse practitioners and/or midwives) regarding physical health concerns in the past month. Less than a third (28\%) received prenatal classes. As well, despite most (84\%) living on low income, less than a third (29\%) reported receiving social benefits such as income assistance or other BC or Canadian entitlements. More girls compared to young women received primary healthcare ( $80 \%$ versus $74 \%$ ) and prenatal classes (32\% versus $25 \%$ ) in the past month; whereas, more young women (37\%) compared to girls (20\%) received income assistance. See Table 5.

\section{Cumulative disadvantage}

Almost all participants (96\%) were experiencing two or more indicators of adversity. As well, more than two thirds (70\%) were experiencing four or more indicators. See Table 6.

Table 2 Baseline socioeconomic disadvantage according to eligibility criteria

\begin{tabular}{|c|c|c|c|c|}
\hline & \multirow[b]{2}{*}{$\begin{array}{l}\text { Total } \\
N=739\end{array}$} & \multicolumn{2}{|l|}{ Age Group } & \multirow[t]{3}{*}{$p$-value } \\
\hline & & $\begin{array}{l}14-19 \text { years } \\
N=361\end{array}$ & $\begin{array}{l}20-24 \text { years } \\
N=378\end{array}$ & \\
\hline & $n(\%)$ & $n(\%)$ & $n(\%)$ & \\
\hline $\begin{array}{l}\text { Low income } \\
\text { (living on }<\$ 20,000 \text { annually) }\end{array}$ & $606 / 726(83.5)$ & $319 / 354(90.1)$ & 287/372 (77.2) & $<0.001$ \\
\hline $\begin{array}{l}\text { Limited education } \\
\text { (no high school completion) }\end{array}$ & $384 / 738(52.0)$ & $246 / 360(68.3)$ & $138 / 378(36.5)$ & $<0.001$ \\
\hline $\begin{array}{l}\text { Preparing to parent while single } \\
\text { (not married or common-law) }\end{array}$ & 670/736 (91.0) & $312 / 360(86.7)$ & $358 / 376(95.2)$ & $<0.001$ \\
\hline
\end{tabular}

Results in bold: $p$-value $<0.05$ 
Table 3 Sociodemographic characteristics, psychological resources and cognitive functioning

\begin{tabular}{|c|c|c|c|c|}
\hline & \multirow[b]{2}{*}{$\begin{array}{l}\text { Total } \\
N=739\end{array}$} & \multicolumn{2}{|l|}{ Age Group } & \multirow[t]{3}{*}{$p$-value } \\
\hline & & $\begin{array}{l}14-19 \text { years } \\
N=361\end{array}$ & $\begin{array}{l}20-24 \text { years } \\
N=378\end{array}$ & \\
\hline & $n(\%)$ & $n(\%)$ & $n(\%)$ & \\
\hline \multicolumn{5}{|l|}{ Sociodemographic Characteristics } \\
\hline Cultural background $^{\mathrm{a}}$ & $n=738$ & $n=361$ & $n=378$ & 0.099 \\
\hline White & $418(56.6)$ & $193(53.5)$ & $225(59.5)$ & \\
\hline Indigenous including First Nations, Métis and Inuit & $79(10.7)$ & $36(10.0)$ & $43(11.4)$ & \\
\hline Indigenous including First Nations, Métis and Inuit and Other & $121(16.4)$ & $73(20.2)$ & $48(12.7)$ & \\
\hline Mixed Heritage $\geq 2$ & $55(7.4)$ & $30(8.3)$ & $25(6.6)$ & \\
\hline Asian (Chinese, S. Asian, or Other) & $32(4.3)$ & $14(3.9)$ & $18(4.8)$ & \\
\hline Other (e.g., Latin-American, Black) & $34(4.6)$ & $15(4.2)$ & $19(5.0)$ & \\
\hline English as first language & $686(93.0)$ & $341(94.7)$ & $345(91.3)$ & 0.091 \\
\hline Highest educational qualification & $n=738$ & $n=360$ & $n=378$ & $<0.001$ \\
\hline Less than high school & $384(52.0)$ & $246(68.3)$ & $138(36.5)$ & \\
\hline High school or equivalent & $270(36.6)$ & $103(28.6)$ & $167(44.2)$ & \\
\hline College or university degree & $84(11.4)$ & $11(3.1)$ & $73(19.3)$ & \\
\hline Income from employment (annual CAD) & $n=726$ & $n=354$ & $n=372$ & $<0.001$ \\
\hline Less than $\$ 5000$ & $308(42.4)$ & $203(57.3)$ & $105(28.2)$ & \\
\hline$\$ 5000-9999$ & $118(16.3)$ & $60(16.9)$ & $58(15.6)$ & \\
\hline$\$ 10,000-19,999$ & $180(24.8)$ & $56(15.8)$ & $124(33.3)$ & \\
\hline$\$ 20,000-29,999$ & $75(10.3)$ & $21(5.9)$ & $54(14.5)$ & \\
\hline$\$ 30,000$ or more & $45(6.2)$ & $14(4.0)$ & $31(8.3)$ & \\
\hline Current Housing & $n=725$ & $n=357$ & $n=368$ & 0.138 \\
\hline House, apartment or condominium & $681(93.9)$ & $332(93.0)$ & $349(94.8)$ & \\
\hline Group home, shelter or foster home & $18(2.5)$ & $13(3.6)$ & $5(1.4)$ & \\
\hline \multirow[t]{2}{*}{ Other (e.g., mobile home/trailer, single-room occupancy residence) } & $26(3.6)$ & $12(3.4)$ & $14(3.8)$ & \\
\hline & Mean (SD) & Mean (SD) & Mean (SD) & \\
\hline Income from employment (annual CAD) & 9928 (10575) & $6811(8976)$ & $12,886(11125)$ & $<0.001$ \\
\hline Age & $19.76(2.36)$ & $17.73(1.17)$ & $21.69(1.40)$ & $<0.001$ \\
\hline \multicolumn{5}{|l|}{ Psychological Resources } \\
\hline Self-Efficacy & $32.28(3.93)$ & $32.08(3.79)$ & $32.47(4.05)$ & 0.179 \\
\hline Mastery & $21.42(3.06)$ & $21.53(3.08)$ & $21.32(3.05)$ & 0.361 \\
\hline \multicolumn{5}{|l|}{ Cognitive Functioning } \\
\hline Shipley 2 - Vocabulary & $24.35(5.12)$ & $23.53(4.76)$ & $25.13(5.33)$ & $<0.001$ \\
\hline Shipley 2 - Abstraction & $11.89(3.65)$ & $11.73(3.57)$ & $12.04(3.73)$ & 0.248 \\
\hline \multicolumn{5}{|l|}{ Executive functioning } \\
\hline Stroop Colour-Word Task Score & $43.99(9.19)$ & $42.46(8.64)$ & $45.45(9.46)$ & $<0.001$ \\
\hline Stroop Interference Score & $5.39(6.72)$ & $4.48(6.15)$ & $6.26(7.12)$ & $<0.001$ \\
\hline Trail Making Test $\mathrm{B}^{\mathrm{b}}$ & $70.55(32.67)$ & $73.92(33.23)$ & $67.32(31.84)$ & 0.006 \\
\hline Trail Making Test $B / A^{b}$ & $44.89(29.28)$ & $47.81(29.87)$ & $42.07(28.45)$ & 0.008 \\
\hline
\end{tabular}

${ }^{\mathrm{a}}$ Participants could give more than one answer; ${ }^{\mathrm{b}}$ Shorter scores represent better performance on a timed task. Results in bold: $p$-value $<0.05$

\section{Discussion}

Our data have depicted a cohort of pregnant girls and young women in $\mathrm{BC}$, Canada, who were recruited to a trial based on selected indicators of socioeconomic disadvantage (young age, low income, limited education and/or single parenting). Yet the data indicated that all participants selected using these socioeconomic and demographic indicators were also experiencing 
Table 4 Health and social adversities including maltreatment experiences

\begin{tabular}{|c|c|c|c|c|}
\hline & \multirow{3}{*}{$\begin{array}{l}\text { Total } \\
N=739 \\
n(\%)\end{array}$} & \multicolumn{2}{|l|}{ Age Group } & \multirow{3}{*}{$\begin{array}{l}p \text { - } \\
\text { value }\end{array}$} \\
\hline & & \multirow{2}{*}{$\begin{array}{l}14-19 \text { years } \\
N=361 \\
n(\%)\end{array}$} & \multirow{2}{*}{$\begin{array}{l}20-24 \text { years } \\
N=378 \\
n(\%)\end{array}$} & \\
\hline & & & & \\
\hline \multicolumn{5}{|l|}{ Unstable housing } \\
\hline Lifetime homelessness (including currently) & $333 / 716(47.0)$ & $154 / 351(44.0)$ & $179 / 365(49.0)$ & \\
\hline Currently homeless & $22 / 721(3.1)$ & $9 / 351(2.6)$ & $13 / 370(3.5)$ & 0.600 \\
\hline Moved $\geq 3$ times or homeless (past year) & $385 / 731(52.1)$ & $183 / 357(51.3)$ & 202/374 (54.0) & 0.503 \\
\hline \multicolumn{5}{|l|}{ Psychological Distress (past month) } \\
\hline Moderate/severe psychological distress & 235/737 (31.9) & $100 / 360(27.8)$ & 135/377 (35.8) & 0.024 \\
\hline Mental health conditions ${ }^{a}$ & $n=739$ & $n=361$ & $n=378$ & \\
\hline Severe anxiety or depression regularly & $346(46.8)$ & $173(47.9)$ & $173(45.8)$ & 0.608 \\
\hline Diagnosed mental disorder (e.g., bipolar disorder or attention problems) & $160(21.7)$ & $73(20.2)$ & $87(23.0)$ & 0.405 \\
\hline Diagnosed developmental conditions (e.g., autism spectrum or learning disorders) & $83(11.2)$ & $35(9.7)$ & $48(12.7)$ & 0.240 \\
\hline \multicolumn{5}{|l|}{ Prenatal substance use } \\
\hline Any cannabis, alcohol or street drug use (past month) ${ }^{a}$ & $172 / 732(23.5)$ & $80 / 357(22.4)$ & $92 / 375(24.5)$ & 0.555 \\
\hline Cannabis use (past month) & $155 / 738(21.0)$ & $75 / 360(20.8)$ & $80 / 378(21.2)$ & 0.984 \\
\hline Alcohol use (past month) & $17 / 736(2.3)$ & $6 / 361(1.7)$ & $11 / 375(2.9)$ & 0.367 \\
\hline Street drug use (past month) & $11 / 736(1.5)$ & $<5 / 358(<2)$ & $7 / 378(1.9)$ & 0.605 \\
\hline Nicotine/cigarette use (past 48 h) & 196/736 (26.6) & $96 / 360(26.7)$ & 100/376 (26.6) & $>0.999$ \\
\hline Second-hand smoke exposure (past week) & 292/736 (39.7) & 150/361(41.6) & 142/375 (37.9) & 0.344 \\
\hline Serious long-term physical health conditions ${ }^{a} n=739$ & & $n=361$ & $n=378$ & \\
\hline Iron-deficiency anemia & $151(20.4)$ & $69(19.1)$ & $82(21.7)$ & 0.437 \\
\hline Asthma or allergies (regular use of puffers) & $139(18.8)$ & $64(17.7)$ & 75 (19.8) & 0.522 \\
\hline Migraines (weekly or more) & $108(14.6)$ & $57(15.8)$ & $51(13.5)$ & 0.436 \\
\hline Serious injury (head/leg) that left a disability & $57(7.7)$ & $24(6.6)$ & $33(8.7)$ & 0.356 \\
\hline Thyroid disease & $21(2.8)$ & $6(1.7)$ & $15(4.0)$ & 0.096 \\
\hline Cardiovascular disease (including high blood pressure) & $13(1.8)$ & $<5(<2)$ & $9(2.4)$ & 0.300 \\
\hline Epilepsy or seizures (weekly or more) & $13(1.8)$ & $7(1.9)$ & $6(1.6)$ & 0.933 \\
\hline Other (e.g., arthritis, irritable bowel syndrome, autoimmune disorders) & $126(17.1)$ & $61(16.9)$ & $65(17.2)$ & 0.992 \\
\hline \multicolumn{5}{|l|}{ Maltreatment experiences ${ }^{\mathrm{a}}$} \\
\hline \multicolumn{5}{|l|}{ Maltreatment at age 16 years or younger } \\
\hline Moderate/severe neglect, physical abuse, emotional abuse and/or sexual abuse & $410 / 728(56.3)$ & $196 / 355(55.2)$ & 214/373 (57.4) & 0.608 \\
\hline \multicolumn{5}{|l|}{ Intimate partner violence in past year } \\
\hline Any physical abuse, emotional abuse and harassment & $363 / 734(49.5)$ & $181 / 358(50.6)$ & $182 / 376(48.4)$ & 0.61 \\
\hline
\end{tabular}

aparticipants could give more than one answer. Results in bold: $p$-value $<0.05$

substantial additional health and social adversities. These added adversities included: housing instability, mental and physical health problems including prenatal substance use, maltreatment during childhood, and IPV exposure recently. As well, despite BC's existing social services, less than a third had received recent income assistance entitlements. Perhaps most telling, almost all were experiencing two or more indicators of adversity while more than two thirds were experiencing four or more - suggesting considerable cumulative disadvantage.
How does this cohort compare to other British Columbians and Canadians? Beyond the eligibility criteria, while directly comparable data were not available for all variables, our cohort nevertheless reported much higher rates of associated health and social adversities including: homelessness and unstable housing, mental health problems including prenatal substance use, and serious physical health problems [43-50]. Rates of child maltreatment and IPV exposure were also twice those found for other Canadians [51, 52]. These comparisons confirm that we recruited a cohort who was 
Table 5 Receiving health and social services ${ }^{a}$

\begin{tabular}{|c|c|c|c|c|}
\hline & \multirow[b]{2}{*}{$\begin{array}{l}\text { Total } \\
N=739\end{array}$} & \multicolumn{2}{|l|}{ Age Group } & \multirow[t]{3}{*}{$p$-value } \\
\hline & & $\begin{array}{l}14-19 \text { years } \\
N=361\end{array}$ & $\begin{array}{l}20-24 \text { years } \\
N=378\end{array}$ & \\
\hline & $n(\%)$ & $n(\%)$ & $n(\%)$ & \\
\hline \multicolumn{5}{|l|}{ Health services for physical health } \\
\hline Primary healthcare (past month) & $567 / 739(76.7)$ & 289/361 (80.1) & 278/378 (73.5) & 0.045 \\
\hline Prenatal classes (past month) & $210 / 739(28.4)$ & $116 / 361(32.1)$ & $94 / 378(24.9)$ & 0.035 \\
\hline \multicolumn{5}{|l|}{ Social services received } \\
\hline Income assistance (past month) & 212/739 (28.7) & $71 / 361(19.7)$ & 141/378 (37.3) & $<0.001$ \\
\hline
\end{tabular}

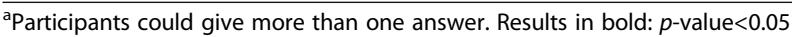

experiencing marked disadvantage, the population NFP is most intended to benefit. See Table 7.

What have our data added? We have shown for the first time that high levels of disadvantage exist for some young Canadians - despite this country's high-income status and its longstanding commitment to equity in access to universal healthcare and related social services [54]. As well, despite provincial/territorial variations in the delivery of health and social programs [53], our data nevertheless have national implications. In Canada, approximately 8000 children are born to adolescent mothers each year [43], while approximately 42,000 are born to young mothers (aged 20-24 years), with many of the latter experiencing low income (13\%) and/or single parenthood (7\%) [43, 46]. Reaching these populations and addressing avoidable adversities during early pregnancy - thereby also increasing children's life chances - is a societal imperative $[16,18,20]$. Our data also suggest that public policy remedies must extend beyond public health and healthcare - encompassing social services, such as ensuring adequate housing and incomes, and preventing child maltreatment and IPV as early as possible in the lifespan. We believe that our study

Table 6 Cumulative disadvantage

\begin{tabular}{llll}
\hline & Total & Age Group & \\
\cline { 3 - 4 } & $N=739$ & $\begin{array}{l}14-19 \text { years } \\
N=361\end{array}$ & $\begin{array}{l}20-24 \text { years } \\
N=378\end{array}$ \\
\hline Indicators of disadvantage & $n(\%)$ & $n(\%)$ & $n(\%)$ \\
1 & $26(3.5)$ & $12(3.3)$ & $14(3.7)$ \\
2 & $73(9.9)$ & $27(7.5)$ & $46(12.2)$ \\
3 & $119(16.1)$ & $61(16.9)$ & $58(15.3)$ \\
4 & $140(18.9)$ & $67(18.6)$ & $73(19.3)$ \\
5 & $152(20.6)$ & $68(18.8)$ & $84(22.2)$ \\
6 & $121(16.4)$ & $68(18.8)$ & $53(14.0)$ \\
7 & $77(10.4)$ & $35(9.7)$ & $42(11.1)$ \\
8 & $29(3.9)$ & $21(5.8)$ & $8(2.1)$ \\
\hline
\end{tabular}

therefore provides new data underscoring an urgent call to action across public sectors not only in $\mathrm{BC}$, but also in Canada.

Our data also have implications for children's rights. We found less than a third of participants had recently received social service entitlements such as income assistance, while approximately half reported recent unstable housing and lifetime homelessness as well as exposure to child maltreatment and IPV. Addressing these serious avoidable adversities is a priority, especially for pregnant youth $[1,4,7,11,54]$. According to international child rights' conventions, $\mathrm{BC}$ and other provinces/territories also have obligations to ensure that the basic needs of all minors are met, including protecting young people from harm and ensuring adequate housing, income and parental/caregiver supports [55, 56]. Our data suggest that these fundamental obligations may not be being fulfilled in BC.

Regarding age differences, we found that in this cohort, young women were facing adversities that were comparable to girls. The statistically significant differences that we did observe between the two groups may be explained by developmental stage (differences in cognitive functioning), eligibility criteria (being single was only a criteria for young women), or developmental context (lower reported income and education may be expected for the pregnant girls who may still be in school and less likely to be employed). The higher proportion of girls accessing primary healthcare and prenatal classes may be a result of better provision of services for these pregnant adolescents compared with young women, although further data are needed. Yet overall, our data suggest that the well-established risks facing children born to adolescents may also extend to children of young women who are experiencing socioeconomic disadvantage in $\mathrm{BC}$.

The BCHCP RCT is embedded within BC's health system, with NFP being delivered as an enhanced public health service - an example of delivering services proportionate to need [18]. Participants will be followed 
Table 7 Indicators of adversity in BCHCP cohort compared to other Canadians

\begin{tabular}{|c|c|c|c|c|}
\hline & \multicolumn{2}{|c|}{ BCHCP population } & \multicolumn{2}{|c|}{ Canadian samples (\%) } \\
\hline & $\begin{array}{l}\text { 14-19years } \\
(\%)\end{array}$ & $\begin{array}{l}\text { 20-24 years } \\
(\%)\end{array}$ & $\%$ & \\
\hline \multicolumn{5}{|l|}{$\mathrm{BCHCP}$ screening criteria } \\
\hline Young age during pregnancy & 49 & 51 & $2-11$ & $\begin{array}{l}\text { Canadian females } \leq 19 \text { years }(2 \%) \text { and } 20-24 \text { years }(11 \%) \\
\text { [43] }\end{array}$ \\
\hline Low income $(<\$ 20,000$ per year $)$ & 90 & 77 & $\begin{array}{l}13- \\
15\end{array}$ & $\begin{array}{l}\text { Canadian females }<18 \text { years }(15 \%) \text { and } \geq 18 \text { years }(13 \%) \\
\text { [44] }\end{array}$ \\
\hline Limited education (no high school completion) & 69 & 37 & 17 & BC females of all ages [45] \\
\hline $\begin{array}{l}\text { Preparing to parent while single (not married or } \\
\text { common-law) }\end{array}$ & 87 & 95 & $7-45$ & $\begin{array}{l}\text { Canadian females } \geq 15 \text { years ( } 7 \%) \text { and living on low } \\
\text { income }(45 \%)[46]\end{array}$ \\
\hline \multicolumn{5}{|l|}{ Additional health and social adversities } \\
\hline Homeless ever (including currently) & 44 & 49 & 5 & Canadians (male and female) 15-24 years [47] \\
\hline Unstable housing (moving in past year) & 35 & 33 & 20 & Canadian females $\geq 15-24$ years [47] \\
\hline Psychological distress & 28 & 36 & 30 & Canadian females $\geq 15$ years and low-income [48] \\
\hline Diagnosed mental disorder & 20 & 23 & 15 & Canadian females $\geq 15$ years [48] \\
\hline Prenatal cannabis use & 21 & 21 & 15 & BC females $12-17$ years [49] \\
\hline Prenatal nicotine use & 27 & 27 & 22 & Canadian females $\geq 15$ years and low income [50] \\
\hline Serious physical health problems & $\leq 19$ & $\leq 22$ & 13 & BC females 12-17 years [49] \\
\hline Child maltreatment when $\leq 16$ years & 55 & 57 & 30 & Canadian females $\geq 15$ years [51] \\
\hline $\begin{array}{l}\text { Exposure to intimate partner violence in recent } \\
\text { past }\end{array}$ & 51 & 48 & $\begin{array}{l}22- \\
40\end{array}$ & $\begin{array}{l}\text { Canadian females } 15-19 \text { years }(40 \%) \text { and } 20-24 \text { years } \\
(22 \%)[52]\end{array}$ \\
\hline
\end{tabular}

throughout pregnancy and until their children reach age 2 years (the duration of the NFP program). Additional outcome findings will be available in 2020-2022. The embedded nature of this RCT ensures that findings are shared quickly and efficiently with policy and practice partners to inform ongoing strategies to better reach populations in need.

There are nevertheless limitations to the data reported here. This cohort was not a representative sample nor did it represent all the potentially eligible girls and young women, in that many (two thirds) were not reached through $\mathrm{BCHCP}$ recruitment efforts. Further collaborative research-practice-policy efforts are needed to better identify and provide services and supports for this "need-to-reach" population. We also acknowledge that the data on education levels does not account for those girls who were still attending high-school $(n=182$ or $25 \%$ of the total cohort). However, all girls were pregnant and preparing to parent at a young age such that their education and employment opportunities were interrupted, placing them and their children at risk for disadvantage.

\section{Conclusions}

Our data suggest that unacceptably high levels of socioeconomic disadvantage exist for some young British Columbians - despite existing health and social services in a high-income province in a high-income country. Concentrated disadvantage for mothers also places children at risk for a range of adversities and for long-term developmental and mental health problems. Therefore, greater health and social supports and services are warranted for this population - to help them and to help their children.

\section{Abbreviations}

BC: British Columbia; BCHCP: British Columbia Healthy Connections Project; CAD: Canadian dollar; IPV: Intimate partner violence; NFP: Nurse-Family Partnership; RCT: Randomized controlled trial; REB: Research Ethics Board

\section{Acknowledgements}

We thank the participants and the BC public health nurses and supervisors who provided the NFP intervention through the RCT. We also thank our policy and practice collaborators in the BC Ministry of Health, the BC Ministry of Children and Family Development, and the Fraser, Interior, Island and Vancouver Coastal Health Authorities. We acknowledge the local Study Team members who have worked day-to-day implementing this trial. Ronald Barr and Lenora Marcellus are members of the Scientific Team. David Olds and Hui Xie provide consulting. During this study, Michael Boyle and Charlotte Waddell were supported by Canada Research Chairs; Harriet MacMillan was supported by the Chedoke Health Chair in Child Psychiatry; and Andrea Gonzalez was supported by a Canadian Institutes of Health Research New Investigator Award and by an Ontario Ministry of Research, Innovation and Science Early Researcher Award.

\section{Authors' contributions}

NC drafted this manuscript and incorporated suggestions from all authors. $\mathrm{NC}, \mathrm{HM}$ and $\mathrm{CW}$ were responsible for overall trial design, with input from team members. CW obtained the original trial funding and led the development of needed policy relationships. NC, RL and CW were responsible for the day-to-day implementation of the trial and for data 
acquisition and quality. NC, YZ, MB and LM conducted the analysis. All authors, NC, RL, DS, YZ, MB, LM, AG, AG, SJ, LT, CV, HM, CW, contributed to interpreting the findings and revising the manuscript for intellectual content. All authors read and approved the final version of the manuscript.

\section{Funding}

The BC Healthy Connections Project is funded by the BC Ministry of Health with support from the BC Ministry of Children and Family Development. The participating Health Authorities (Fraser, Interior, Island and Vancouver Coastal Health) and the Ministry of Health have funded NFP implementation. The Djavad Mowafaghian and R. and J. Stern Family Foundations have provided generous additional supports. Study design, data collection and data analysis are all being conducted independently of funder involvement. This manuscript was prepared independently and does not necessarily reflect the views of the trial's funders. No funding was provided for the preparation of this manuscript.

\section{Availability of data and materials}

All data supporting the results are included in this article.

\section{Ethics approval and consent to participate}

This study received research ethics board (REB) approvals from all participating organizations: Simon Fraser University (2012 s0738); the University of British Columbia and Fraser Health Authority (H13-01938); the University of Victoria (13-277); McMaster University (13-570); the Public Health Agency of Canada (2012-0039); and Interior Health (2012-13-014-E), Island Health (BC2013-069) and Vancouver Coastal Health (V13-01938) Authorities. All participants provided written informed consent prior to data collection commencing. The participating research ethics review boards waived parental consent requirement for pregnant minors. Specifically, we received REB approvals for all participants who were pregnant minors (aged 18 years or younger in $\mathrm{BC}$ ) to provide informed consent to participate; these younger participants were deemed to be competent minors by our REBs. However, parental consent and participant assent was obtained whenever a referring public health nurse deemed a minor as not competent to provide informed consent (for example, due to learning disability).

\section{Consent for publication}

Not applicable.

\section{Competing interests}

The authors declare that they have no competing interests.

\begin{abstract}
Author details
${ }^{1}$ Children's Health Policy Centre, Faculty of Health Sciences, Simon Fraser University, Room 2431, 515 West Hastings Street, Vancouver, BC V6B 5K3, Canada. ${ }^{2}$ Offord Centre for Child Studies, Faculty of Health Sciences, McMaster University, Hamilton, Ontario, Canada. ${ }^{3}$ Faculty of Health Sciences, Simon Fraser University, Burnaby, BC, Canada. ${ }^{4}$ Faculty of Health Sciences, McMaster University, Hamilton, Ontario, Canada. ${ }^{5}$ School of Nursing, McMaster University, Hamilton, Ontario, Canada. ${ }^{6}$ Public Health Agency of Canada, Ottawa, Ontario, Canada. ${ }^{7}$ School of Nursing, University of BC, Vancouver, BC, Canada. ${ }^{8}$ Departments of Psychiatry and Behavioural Neurosciences and of Pediatrics, Offord Centre for Child Studies, Faculty of Health Sciences, McMaster University, Hamilton, Ontario, Canada.
\end{abstract}

\section{Received: 15 February 2019 Accepted: 13 August 2019}

Published online: 22 August 2019

\section{References}

1. Aizer A, Currie J. The intergenerational transmission of inequality: maternal disadvantage and health at birth. Science. 2014;344:856-61.

2. Evans G. Childhood poverty and adult psychological well-being. Proc Natl Acad Sci U S A. 2016;113(52):14949.

3. Reiss F. Socioeconomic inequalities and mental health problems in children and adolescents: a systematic review. Soc Sci Med. 2013;90:24-31.

4. Luong M. Life after teenage motherhood. Perspectives on labour and income. Ottawa: Statistics Canada; 2008.

5. Chico E, Gonzalez A, Ali N, Steiner M, Fleming AS. Executive function and mothering: challenges faced by teenage mothers. Dev Psychobiol. 2014;56: $1027-35$.
6. Ekéus C, Christensson K, Hjern A. Unintentional and violent injuries among pre-school children of teenage mothers in Sweden: a national cohort study. J Epidemiol Community Health. 2004;58:680-5.

7. Jutte DP, Roos NP, Brownell MD, Briggs G, MacWilliam L, Roos LL. The ripples of adolescent motherhood: social, educational, and medical outcomes for children of teen and prior teen mothers. Acad Pediatr. 2010; 10:293-301.

8. Putnam-Hornstein E, Cederbaum JA, King B, Eastman AL, Trickett PK. A population-level and longitudinal study of adolescent mothers and intergenerational maltreatment. Am J Epidemiol. 2015;181:496-503.

9. Gilbride SJ, Wild C, Wilson DR, Svenson LW, Spady DW. Socio-economic status and types of childhood injury in Alberta: a population based study. BMC Pediatr. 2006;6:e1-e10. https://doi.org/10.1186/1471-2431-6-30.

10. Lefebvre R, Fallon B, Van Wert M, Filippelli J, et al. Behav Sci. 2017;7:e1-e12. https://doi.org/10.3390/bs7010006.

11. Oh DL, Jerman P, Silvério Marques S, Koita K, Purewal Boparai SK, Burke Harris N, et al. Systematic review of pediatric health outcomes associated with childhood adversity. BMC Pediatr. 2018;18:e1-e19. https://doi.org/10.11 86/s12887-018-1037-7.

12. Orton E, Kendrick D, West J, Tata $\amalg$. Independent risk factors for injury in pre-school children: three population-based nested case-control studies using routine primary care data. PLoS One. 2012;7:e1-8. https://doi.org/1 0.1371/journal.pone.0035193.

13. Goodman SH, Rouse MH, Connell AM, Broth MR, Hall CM, Heyward D. Maternal depression and child psychopathology: a meta-analytic review. Clin Child Fam Psychol Rev. 2011;14:1-27.

14. Grant KS, Petroff R, Isoherranen N, Stella N, Burbacher TM. Cannabis use during pregnancy: pharmacokinetics and effects on child development. Pharmacol Ther. 2018;182:133-51.

15. Huang C-C, Vikse JH, Lu S, Yi S. Children's exposure to intimate partner violence and early delinquency. J Fam Violence. 2015;30:953-65.

16. World Health Organization and Calouste Gulbenkian Foundation. Social determinants of mental health. Geneva: World Health Organization; 2014.

17. Braveman P, Egerter S, Williams DR. The social determinants of health: coming of age. Annu Rev Public Health. 2011;32:381-98.

18. Marmot M, Allen J, Goldblatt P, Boyce T, McNeish D, Grady M, et al. Fair society, healthy lives. London: Institute of Health Equity; 2010.

19. Heckman JJ. Skill formation and the economics of investing in disadvantaged children. Science. 2006;312:1900-2.

20. Waddell C, Schwartz C, Andres C. Making children's mental health a public policy priority: for the one and the many. Public Health Ethics. 2018;11:191-200.

21. MacMillan HL, Wathen CN, Barlow J, Fergusson DM, Leventhal JM, Taussig $\mathrm{HN}$. Interventions to prevent child maltreatment and associated impairment. Lancet. 2009;373:250-66.

22. Hertzman C, Boyce T. How experience gets under the skin to create gradients in developmental health. Annu Rev Public Health. 2010;31:329-47.

23. Olds DL. Preventing child maltreatment and crime with prenatal and infancy support of parents: the nurse-family partnership. J Scand Stud Criminol Crime Prev. 2008;9:2-24.

24. Bonevski B, Randell M, Paul C, Chapman K, Twyman L, Bryant J, et al. Reaching the hard-to-reach: a systematic review of strategies for improving health and medical research with socially disadvantaged groups. BMC Med Res Methodol. 2014;14:e1-e29. https://doi.org/10.1186/1471-2288-14-42.

25. Catherine NLA, Gonzalez A, Boyle M, Sheehan D, Jack SM, Hougham KA, et al. Improving children's health and development in British Columbia through nurse home visiting: a randomized controlled trial protocol. BMC Health Serv Res. 2016;16:e1-e13. https://doi.org/10.1186/s12913-016-1594-0.

26. Robling M, Bekkers MJ, Bell K, Butler CC, Cannings-John R, Channon S, et al. Effectiveness of a nurse-led intensive home-visitation programme for firsttime teenage mothers (building blocks): a pragmatic randomised controlled trial. Lancet. 2016:387:146-55.

27. Mejdoubi J, van den Heijkant SC, van Leerdam FJ, Crone M, Crijnen A, HiraSing RA. Effects of nurse home visitation on cigarette smoking, pregnancy outcomes and breastfeeding: a randomized controlled trial. Midwifery. 2014;30:688-95.

28. Statistics Canada. 2006 Census of Population. 2010. https://www12.statcan. gc.ca/census-recensement/2006/index-eng.cfm.

29. Schwarzer R, Jerusalem M. Generalized self-efficacy scale. In: Weinman J, Wright S, Johnston M, editors. Measures in health psychology: a user's portfolio. Windsor: Nfer-Nelson; 1995. p. 35-7. 
30. Pearlin LI, Schooler C. The structure of coping: erratum. J Health Soc Behav. 1978;19:237.

31. Shipley W, Gruber C, Martin T, Klein A. Shipley-2. Los Angeles: Western Psychological Services; 2009.

32. Golden C. Stroop color and word test: cat. No. 30150M. In: a manual for clinical and experimental uses. Chicago: Stoelting; 1978.

33. Reitan R, Wolfson D. The Halstead-Reitan Neurospychological test battery. 2nd ed. Tuscon: Neuropsychology Press; 1993

34. Sánchez-Cubillo I, Periáñez JA, Adrover-Roig D, Rodríguez-Sánchez JM, RíosLago M, Tirapu J, et al. Construct validity of the trail making test: role of task-switching, working memory, inhibition/interference control, and visuomotor abilities. J Int Neuropsychol Soc. 2009;15:438-50.

35. Gaetz S, Barr C, Friesen A, Harris B, Hill C, Kovacs-Burns K, et al. Canadian definition of homelessness. Toronto: Canadian Observatory on Homelessness Press; 2012.

36. Greater Vancouver Regional Steering Committee on Homelessness. Results of the 2014 homeless count in the Metro Vancouver Region. Vancouver: Greater Vancouver Regional Steering Committee on Homelessness; 2014.

37. Bernstein DP, Stein JA, Newcomb MD, Walker E, Pogge D, Ahluvalia T, et al. Development and validation of a brief screening version of the childhood trauma questionnaire. Child Abuse Negl. 2003;27:169-90.

38. Hegarty K, Bush R, Sheeham M. The composite abuse scale: further development and assessment of reliability and validity of a multidimensional partner abuse measure in clinical settings. Violence Vict. 2005:20:529-47.

39. Kessler RC, Andrews G, Colpe L, Hiripi E, Mroczek DK, Normand S-LT, et al. Short screening scales to monitor population prevalences and trends in non-specific psychological distress. Psychol Med. 2002;32:959-76.

40. Statistics Canada. Canadian community health survey annual component. 2011. http://www23.statcan.gc.ca/imdb/p2SV.pl?Function=getSurvey\&SDDS= 3226\&lang $=e n \& d b=i m d b \& a d m=8 \& d i s=2$.

41. Statistics Canada. Canadian health measures survey (Cycle 2). 2012. http:// www23.statcan.gc.ca/imdb/p2SV.pl?Function=getSurvey\&Survld=12954 $8 \&$ Instald $=62444 \&$ SDDS $=5071$.

42. Statistics Canada. National Longitudinal Survey of Children and Youth (Cycle 8). 2009. http://www23.statcan.gc.ca/imdb-bmdi/document/4450_D2_T9_V4-eng.

43. Statistics Canada. Live births, by age of mother. 2018. https://www150. statcan.gc.ca/t1/tbl1/en/tv.action?pid=1310041601.

44. Statistics Canada. Low income statistics by age, sex and economic family type, Table 11-10-0135-01. 2019. https://www150.statcan.gc.ca/t1/tbl1/en/cv. action?pid=1110013501.

45. Statistics Canada. Education indicators in Canada: An international perspective. 2017. https://www150.statcan.gc.ca/n1/pub/81-604-x/2017001/ t/tblc2.3-eng.htm.

46. Urquia ML, O'Campo PJ, Ray JG. Marital status, duration of cohabitation, and psychosocial well-being among childbearing women: a Canadian nationwide survey. Am J Public Health. 2013;103:e8-e15. https://doi.org/1 0.2105/AJPH.2012.301116.

47. Rodrigue S. Hidden homelessness in Canada. Ottawa: Statistics Canada; 2016

48. Caron J, Liu A. A descriptive study of the prevalence of psychological distress and mental disorders in the Canadian population: comparison between low-income and non-low-income populations. Chronic Dis Can. 2010;30:84-94

49. Smith A, Saewyc E, Forsyth K, Poon C, Peled M, Martin S, et al. Balance and connection in BC: the health and well-being of our youth. Results of the 2018 BC adolescent health survey. Vancouver: McCreary Centre Society; 2019.

50. Al-Sahab B, Saqib M, Hauser G, Tamim H. Prevalence of smoking during pregnancy and associated risk factors among Canadian women: a national survey. BMC Pregnancy Childbirth. 2010;10:e1-e10. https://doi.org/10.11 86/1471-2393-10-24.

51. Afifi TO, MacMillan HL, Boyle M, Taillieu T, Cheung K, Sareen J. Child abuse and mental disorders in Canada. CMAJ. 2014;186:e324-32. https://doi.org/1 0.1503/cmaj.131792.

52. Daoud N, Urquia ML, O'Campo P, Heaman M, Janssen PA, Smylie J, et al. Prevalence of abuse and violence before, during, and after pregnancy in a national sample of Canadian women. Am J Public Health. 2012;102:1893-901.

53. Waddell C, Georgiades K, Duncan L, Comeau J, Reid GJ, O'Briain W, et al. The 2014 Ontario child health study findings: policy implications for Canada. Can J Psychiatr. 2019;64:227-31.

54. Allen J, Balfour R, Bell R, Marmot M. Social determinants of mental health. Int Rev Psychiatry. 2014;26:392-407.
55. British Columbia Government. Child, Family and Community Service Act. Victoria: British Columbia Government; 1996.

56. United Nations. Convention on the Rights of the Child. Geneva: United Nations; 1989

\section{Publisher's Note}

Springer Nature remains neutral with regard to jurisdictional claims in published maps and institutional affiliations.
Ready to submit your research? Choose BMC and benefit from:

- fast, convenient online submission

- thorough peer review by experienced researchers in your field

- rapid publication on acceptance

- support for research data, including large and complex data types

- gold Open Access which fosters wider collaboration and increased citations

- maximum visibility for your research: over $100 \mathrm{M}$ website views per year

At BMC, research is always in progress.

Learn more biomedcentral.com/submissions 